\title{
Synthesis and Fluorescence Spectrum of N, N' Bis (2-Hydroxy Benzylidene) Benzidine with Different Solvents and Different PH
}

\author{
Zeyad A. Saleh, Dhaidan Kh. Kafi* \\ Physics Department, College of Science, AL-Mustansiriyah University, Baghdad, Iraq
}

Email address:

dhkh77@yahoo.com (D. Kh. Kafi)

${ }^{*}$ Corresponding author

\section{To cite this article:}

Zeyad A. Saleh, Dhaidan Kh. Kafi. Synthesis and Fluorescence Spectrum of N, N' Bis (2-Hydroxy Benzylidene) Benzidine with Different Solvents and Different PH. American Journal of Optics and Photonics. Vol. 5, No. 4, 2017, pp. 36-44. doi: 10.11648/j.ajop.20170504.11

Received: January 21, 2017; Accepted: February 16, 2017; Published: Octoberh 30, 2017

\begin{abstract}
In this study, a fluorescent compound; N, N'-Bis (2-Hydroxy Benzylidene) Benzidine, $\left(\mathrm{C}_{26} \mathrm{H}_{20} \mathrm{~N}_{2} \mathrm{O}_{2}\right)$ prepared and calculated by FT-IR, ${ }^{1} \mathrm{H}$ NMR, UV-Vis and fluorescence procedures. The ${ }^{1} \mathrm{H}$ NMR field examined in Chloroform-d solvent. The Fourier Transform-Infrared FT-IR in hard case detected in the area 4000-600 cm-1. The absorption spectrum of the Bis (2Hydroxy Benzylidene) Benzidine resolved in tetrahydrofuran registered on the range $250-800 \mathrm{~nm}$. The fluorescence spectrum show in the region $520-700 \mathrm{~nm}$ in different $\mathrm{pH}$ at room temperature. Photoluminescent properties of the title compound examined in tetrahydrofuran, Chloroform, acetone, and Toluene. The intensity and Stoke's move of N, N' Bis (2-Hydroxy Benzylidene) Benzidine in THF solvent found greater than other solvents. The structural value, fundamental vibration modes, Proton Nuclear magnetic resonance isotropic chemical changes, and absorption spectrum of the N, N'-Bis (2-Hydroxy Benzylidene) Benzidine computed by density functional theory (DFT) using B3LYP/6-311G (d, p) basis set.
\end{abstract}

Keywords: Fluorescence, N, N' Bis (2-Hydroxy Benzylidene) Benzidine, Solvent Effect, PH Effect, Density Functional Theory (DFT)

\section{Introduction}

The Fluorescent compounds now overwhelm the line of molecular sensors for chemical studies and biologically on account of them to offer priority to high discovery sensitivity, low noise, and large active domains [1, 2]. These characteristics will be especially apparent in the change of modern optical analytical tools high thoroughness. Fluorescent compounds offering high absorptivity and fluorescence quantum produce, which participate in the cause that they are the greatest significant clan of luminescent analyses [3]. The evolution of luminescence probes and sensors is the topic of dense research both in organic and health science [4]. Fluorescent compounds which syntheses from molecules are strong apparatus to imagine the organic procedure in active cells and creatures. Since detection of aromatic fluorescent composites in the end nineteenth century, endeavours have prepared to "see" the conduct of exact biomolecules in active systems using dye as database. Also, many fluorescent sensors or organic sensors, which acquainted as molecules that offer an alteration in fluorescence ownerships in the presence of their essential molecule studied and applied to biological studying. Presently, a change of sensors is a ready that metallic ions, enzyme liveliness, $\mathrm{pH}$, and sensor molecules [5]. Fluorescence is a strong implement for examining the construction and dynamics of substance or active systems at a molecular field. Solid surfaces, Polymers, proteins, liquid of surfactants, organic membranes, nuclei acids and organism cells are famous samples of systems in which assessment of the factors like polarity, instruction, liquids, electrical potential and molecular mobility is conceivable by incomes fluorescent molecules taking the role of sensors $[6,7,8]$. The many applications of fluorescence compounds confirm the requirement for an account of the fluorescence 
characteristics of this molecule in a liquid solution. Specially, a characterization of the effects of solvent, $\mathrm{pH}$, concentration, irradiation, and excitation wavelength possibly increases the sensitivity and usage of enzyme and other determinations as well as clarify other observations of living and chemical advantage [9]. In this study, the synthesis new fluorescent Schiff base compound by reaction to benzidine and 2-Hydroxy benzylidene. The compound named $\mathrm{N}, \mathrm{N}^{\prime}$ bis (2-hydroxy benzylidene) benzidine synthesized by standard processes and described by the elemental analysis and determination of the melting point (figure 1).

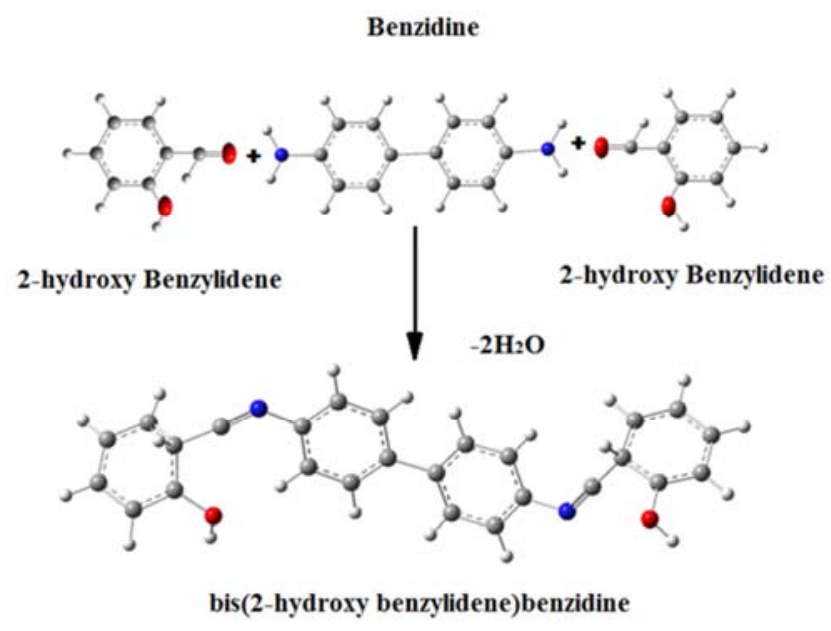

Figure 1. Synthesis of $N, N^{\prime}$ bis (2-hydroxy benzylidene) benzidine.

\section{Materials and Methods}

All Chemical Materials utilized for the preparing of Bis (2hydroxy benzylidene) Benzidine with a stated purity of $99 \%$. Benzidine from fluka chemicals. (2-hydroxy benzylidene) from the Sigma Chemical Company. (USA), the solvents such as benzene, tetrahydrofuran, Ethanol, Chloroform, Toluene and Aceton were of high purity also utilized without additional purification.

Synthesis of Bis (2-hydroxy benzylidene) Benzidine

The Schiff base synthesized from the mixing (tow moles) 2-hydroxy benzylidene, with benzidine (one mole) in $25 \mathrm{ml}$ benzene and (3-4) droplets of glacial acetic acid. This blend refluxed on aqueous baths for more ten hours. A result masses discrete out on cooling, filtered off.

Yield: $75 \%$. Colour: Yellow. Melting Point: $210-212^{\circ} \mathrm{C}$. Molecular formula $\mathrm{C}_{26} \mathrm{H}_{20} \mathrm{~N}_{2} \mathrm{O}_{2}$. FT-IR $\left(\mathrm{KBr}, \mathrm{v}, \mathrm{cm}^{-1}\right)$ : 1616 $(\mathrm{C}=\mathrm{N}), 1568(\mathrm{C}=\mathrm{C}), 3554(\mathrm{OH}), 3057(\mathrm{C}-\mathrm{H})$. UV-Vis $(\mathrm{THF})$ : $\lambda_{\max } / \mathrm{nm}(\log \varepsilon)$ 400. 1H NMR (300 MHz, Chloroform-d6, $\delta$, ppm): 7.2, 7.34-7.76 and $8.68 \mathrm{ppm}$ are the proton signals due to the solvent, protons of aromatic and azomethine $(-\mathrm{CH}=\mathrm{N}-)$ proton one-to-one, the proton signal of phenolic $(-\mathrm{OH})$ proton disappear

\section{Experimental Parts}

The Experimental characterizations of the compound considered by FT-IR, 1H NMR, absorbance spectrum and fluorescence spectrum techniques. Under is an explanation of these techniques.

\subsection{FT-IR Spectrum}

Fourier transform-infrared spectra (FT-IR) calculations were used the $\mathrm{KBr}$ technique utilizing a Shimadzu corporation 8400S FTIR spectrometer. The FT-IR spectrum calculated in the region of $4000-400 \mathrm{~cm}^{-1}$.

\section{2. $U V-V i s$ Spectrum}

All absorbance spectrum of the Bis (2-hydroxy benzylidene) Benzidine studied in the range 200-800 NM is utilizing a Perkin Elmer Lambda $35 \mathrm{UV}-$ Vis spectrometer.

\section{3. ${ }^{1}$ H NMR Spectrum}

${ }^{1} \mathrm{H}$ Proton magnetic resonance spectrum studied (299K) on AV300 MHz spectrometer. The composite resolved in Chloroform-d solvent. Chemical shifts studied in ppm proportional to tetramethylsilane (TMS) for ${ }^{1} \mathrm{H}$ NMR. $1 \mathrm{H}$ Proton magnetic resonance spectrum gained at $300 \mathrm{MHz}$ of the base frequency.

\subsection{Fluorescence Spectrum}

A Shimadzu RF-5301PC spectrofluorophotometer utilized in fluorescence computations. The spectrum of the title compound gained in THF solvent, the concentration was $2 \times 10^{-5} \mathrm{M}$.

\subsection{Computational Systems}

The structural geometry, with no similarity constrains, studied by Gaussian 09 package utilized density functional theory (DFT) computing with a cross functional B3LYP (Becke's three parameter reciprocity functionality integrated with the LYP incline modified association useful) at the foundation set 6-311G (d.p) by the Berny way [13] to study all constant points as a minimum. The computed vibrational frequencies determined that the structure was steady (no fantasy frequency). The TD-DFT way has commonly found a powerful and accurate way of characterizing low-lying excited cases of combined molecules and useful to dissolve many of chemical and physical issues. Based on the studied geometry and by utilizing time-dependent density functional model (TD-DFT) ways in a vacuum, the allowable excitation and oscillator powers of the electronic absorption spectrum prognosticated. Lastly, the Nuclear Magnetic Resonance (NMR) chemical shift performed utilizing Gauge Independent Atomic Orbital (GIAO) way [14, 15].

\section{Results and Discussion}

\subsection{Vibrational Frequencies}

The theoretical FT-IR at DFT/B3LYP level of 6-311G (d, p) basis set of the title compound is given in Figure (2). furthermore, the FT-IR spectrum of the Benzidine and Bis 
(2-hydroxy benzylidene) benzidine are given in Figures (3), (4). The vibrational frequencies of the fundamental types of Bis (2-hydroxy benzylidene) benzidine spotted nearby the theoretical IR frequencies, the IR computations of the title compound are shown in table (1). The omission unharmonicity in a actual system, the vibrational frequency theory for Bis (2-hydroxy benzylidene) benzidine with B3LYP/6-311G $(\mathrm{d}, \mathrm{p})$ are little larger than the studied values.

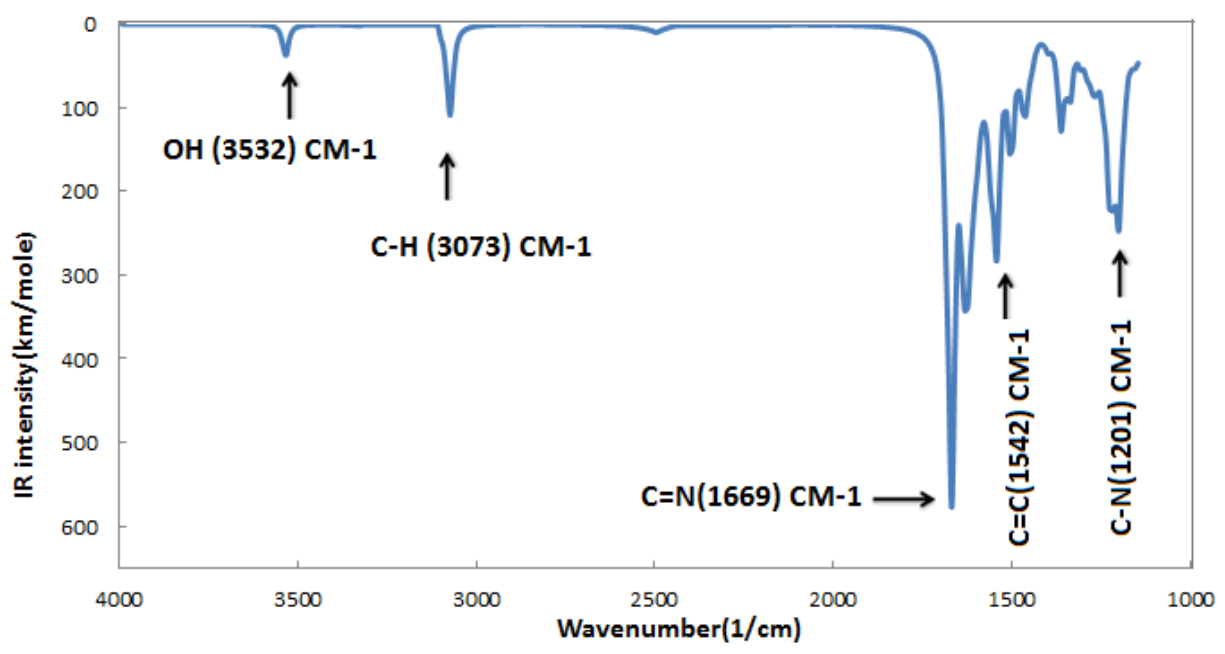

Figure 2. Theoretical FT-IR spectrum of bis (2-hydroxy benzylidene) benzidine.

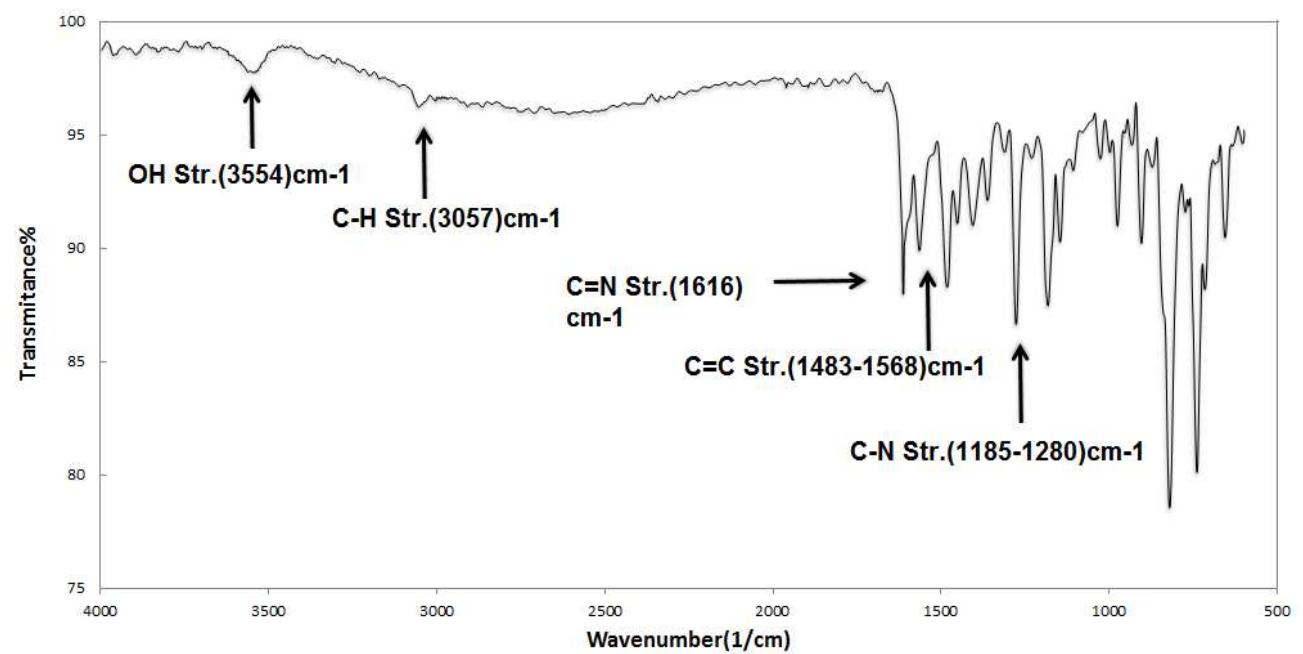

Figure 3. Experimental FT-IR spectrum of bis (2-hydroxy benzylidene) benzidine.

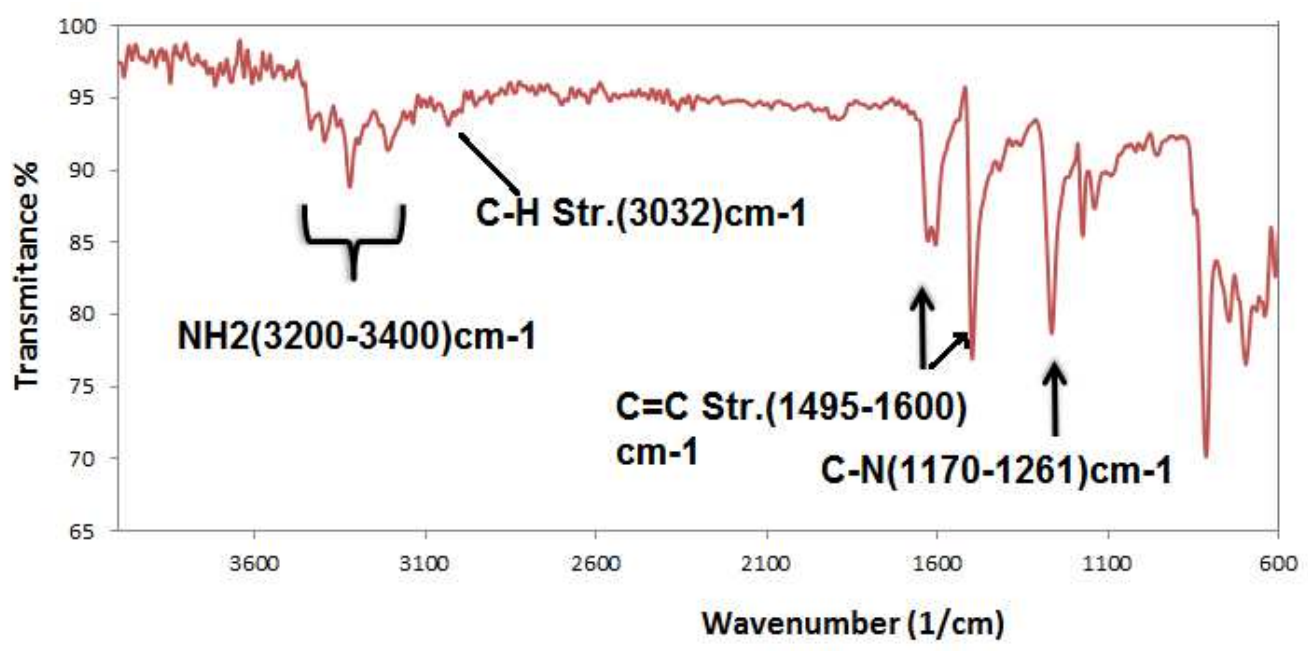

Figure 4. FT-IR spectrum of benzidine. 
Table 1. Theoretical and Experimental FT-IR spectroscopy for bis (2-hydroxy benzylidene) benzidine.

\begin{tabular}{lllllll}
\hline compound & $(\mathbf{O H})$ & $\mathbf{( C = N )}$ & $\mathbf{( C - N )}$ & $\mathbf{( C - H )}$ & $\mathbf{( C = C )}$ & $(\mathbf{N H 2})$ \\
\hline Benzidine & - & - & $1170-1261$ & 3032 & $1495-1600$ & $3200-3400$ \\
Experimental bis (2-hydroxy benzylidene) benzidine & 3554 & 1616 & $1185-1280$ & 3057 & $1483-1568$ & - \\
Theoretical bis (2-hydroxy benzylidene) benzidine & 3532 & 1669 & 1200 & 3073 & 1542 & - \\
\hline
\end{tabular}

\subsection{1. $C-H$ Vibrations}

The aromatic compound displays the vibration modes of $\mathrm{C}-\mathrm{H}$ in the domain $3000-3100 \mathrm{~cm}^{-1}$ [16]. The band of C-H extending vibrations of the bis (2-hydroxy benzylidene) benzidine was $3057 \mathrm{~cm}^{-1}$. DFT calculations predict refer to modes $3073 \mathrm{~cm}^{-1}$ for B3LYP/6-311G $(\mathrm{d}, \mathrm{p})$ levels of the model. The computations presented that the theoretical and experimental data closely coincide and these projects are in decent nearby with literature values [17].

\subsection{2. $C$-C Vibrations}

In Benzene rings the $\mathrm{C}=\mathrm{C}$ stretching modes are appearing in the spectral domain $1650-1400 \mathrm{~cm}^{-1}$ [18]. In the title compound, the stretching vibrations of $\mathrm{C}=\mathrm{C}$ found at 1483$1568 \mathrm{~cm}^{-1}$. These calculations agree with theoretical way and in addition to nearby with the literature values [17].

\subsection{3. $C=N$ Vibrations}

The new peak appears at $1616 \mathrm{~cm}^{-1}$ after the condensation reaction indicating amine $(\mathrm{CH}=\mathrm{N})$ bond. $-\mathrm{NH}_{2}$ peaks of benzidine vanish in the FT-IR spectrum of Bis (2-hydroxy benzylidene) benzidine. This result agrees with the studied vibrations $1669 \mathrm{~cm}^{-1}$ obtained from DFT for B3LYP/6-311G $(\mathrm{d}, \mathrm{p})$. The consequences presented that the theoretical results closely accord with experimental and as well as with the literature data [19].

\subsubsection{OH Group}

The $\mathrm{OH}$ group of alcohols and phenols presents a powerful stretch in the area between 3200 and $3650 \mathrm{~cm}^{-1}$ [17]. In states where hydrogen bonding is present (as it would be in neat fluids, unless it was banned by steric hindrance) this absorption is fairly wide and typically between 3200 and $3600 \mathrm{~cm}^{-1}$ [17, 19]. The IR spectrum of Bis (2-hydroxy benzylidene) benzidine at $3554 \mathrm{~cm}^{-1}$ with strong and medium assigned of the title compound. These results are a good agreement with B3LYP/6- $311 \mathrm{G}(\mathrm{d}, \mathrm{p})$ way and in addition to the literature data [17.19].

\subsection{NMR Spectrum for the N, N Bis (2-Hydroxy Benzylidene) Benzidine}

Experimental ${ }^{1} \mathrm{H}-\mathrm{NMR}$ spectrum of Bis (2-hydroxy benzylidene) benzidine is given in Figure (6). The molecular geometry of Schiff bases established in this spectrum. NMR spectral data abridged as follows: ${ }^{1} \mathrm{H}$ NMR/CDCL3/ $/ \mathrm{ppm}$ : The proton signals of solvent at 7.24ppm., 7.34-7.76 ppm is due to the protons of aromatic and azomethine $(-\mathrm{CH}=\mathrm{N}-)$ proton at $8.68 \mathrm{ppm}$, the proton of phenolic $(-\mathrm{OH})$ is disappear because a protic deuterated solvent is used (e.g., D2O or $\mathrm{CD} 3 \mathrm{OD})$, then the $\mathrm{OH}$ proton will exchange with the deuterium and the peaks will shrink or disappear entirely, since $\mathrm{D}(2 \mathrm{H})$ does not show up in the $1 \mathrm{H}$ NMR spectrum. The theoretical spectrum, which studied with GIAO way using DFT/B3LYP/6-311G (d, p) way agrees with Experimental ${ }^{1} \mathrm{H}$ NMR spectrum, using Gaussian 09 package to perform The NMR spectra calculations, as shown figure (5).

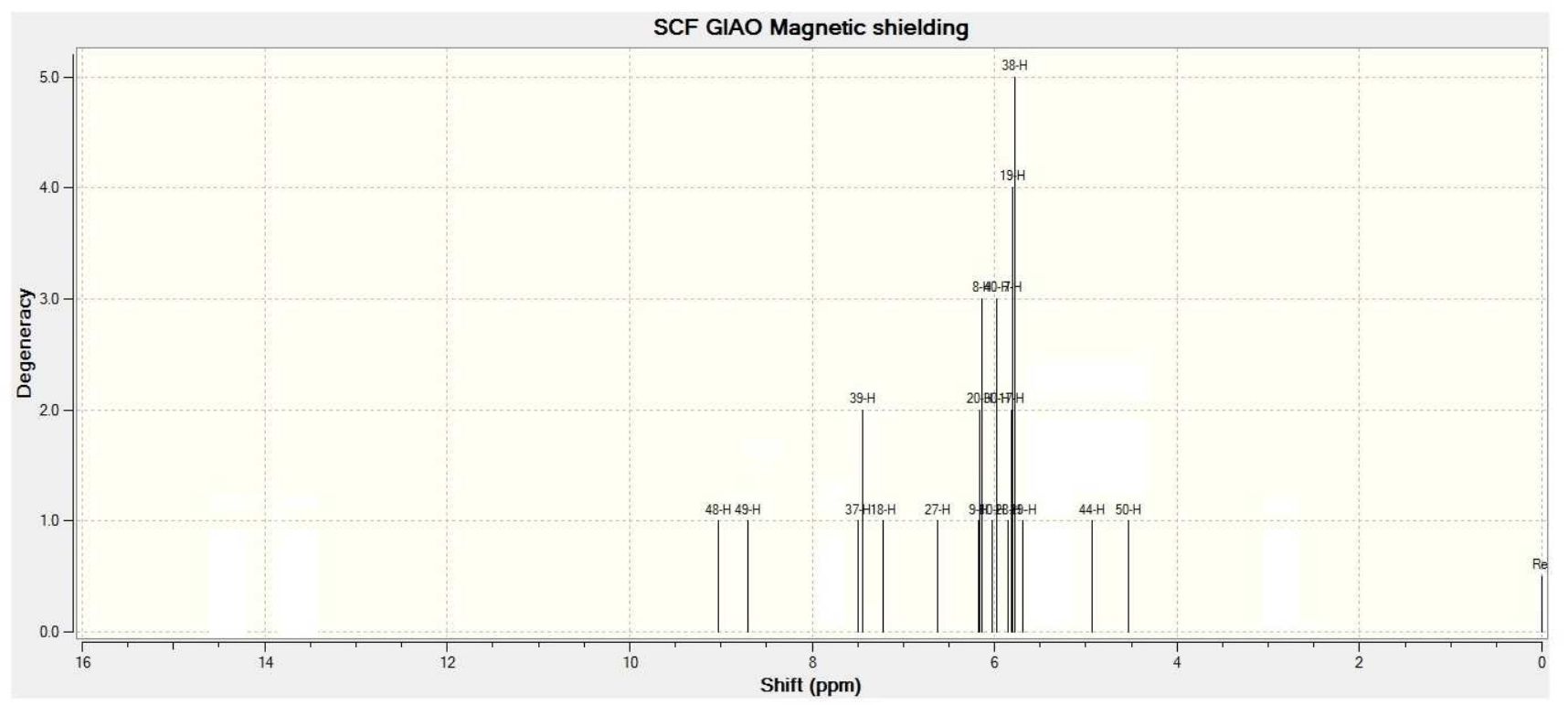

Figure 5. Theoretical ${ }^{l} H$ NMR of Bis (2-hydroxy benzylidene) benzidine compound. 


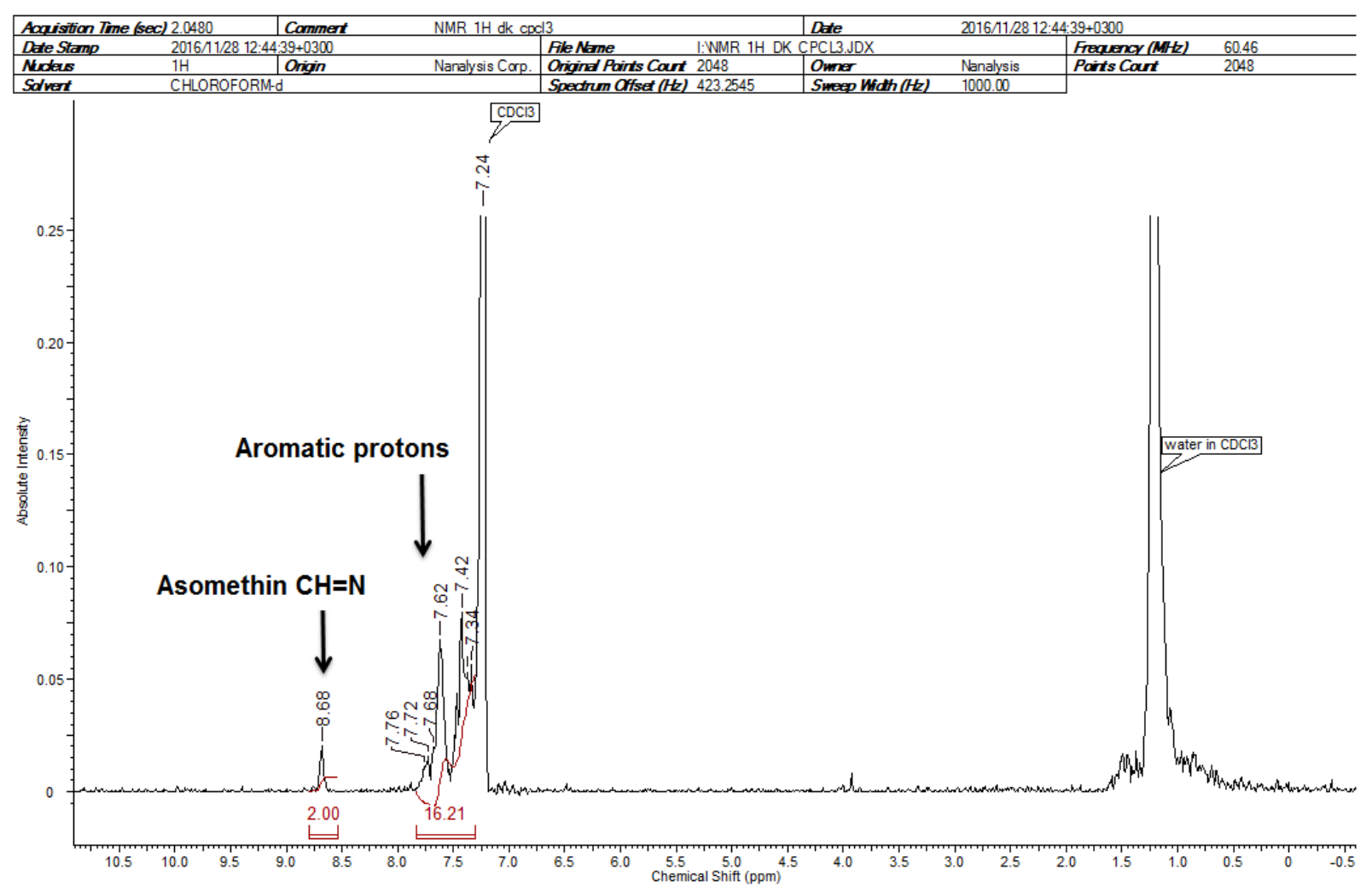

Figure 6. Experimental ${ }^{l} h N M R$ of Bis (2-hydroxy benzylidene) benzidine in $C D C L_{3}$ solvent.

\subsection{UV/Vis Spectrum}

Quantum calculations were approved to the electronic properties of N, N' Bis (2-hydroxy benzylidene) benzidine. The TD-DFT/B3LYP way utlitizing 6-311G $(d, p)$ basis sets utilized to define the excited states for examining the UV-Vis absorption spectrum of the title compound. The experimental and theoretical absorption spectrum of the title compound can be seen in Figures (7), (8). From the figures, UV-Vis spectrum of the title compound displays one peak at $380 \mathrm{~nm}$. From TD-DFT (C-PCM) /B3LYP method, the theoretical absorption bands expected at $400 \mathrm{~nm}$ at $6-311 \mathrm{G}(\mathrm{d}, \mathrm{p})$ basis set and in addition to can show the data are the closest with compared to the experimental data.

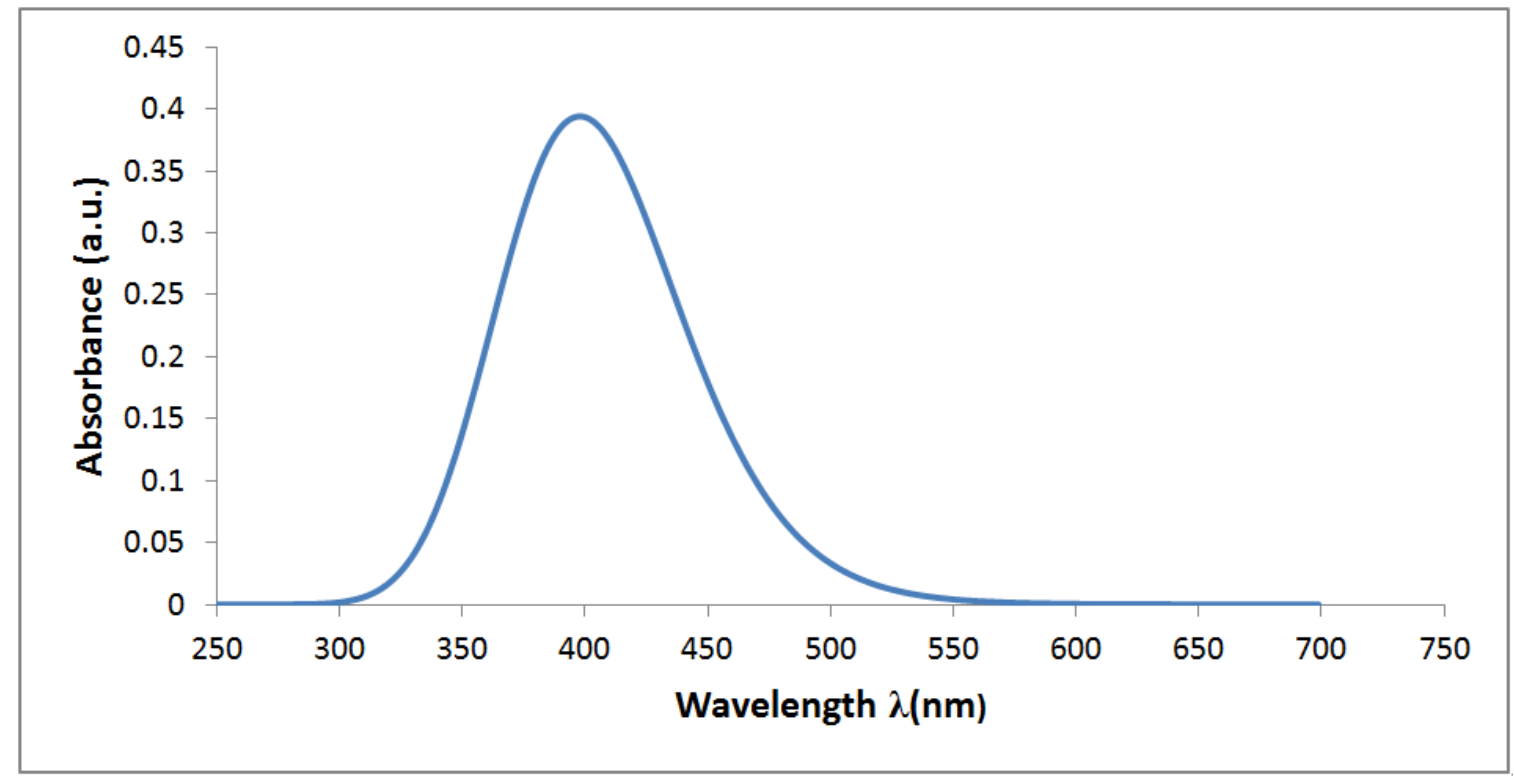

Figure 7. Theoretical electronic spectrum for Bis (2-hydroxy benzylidene) benzidine 


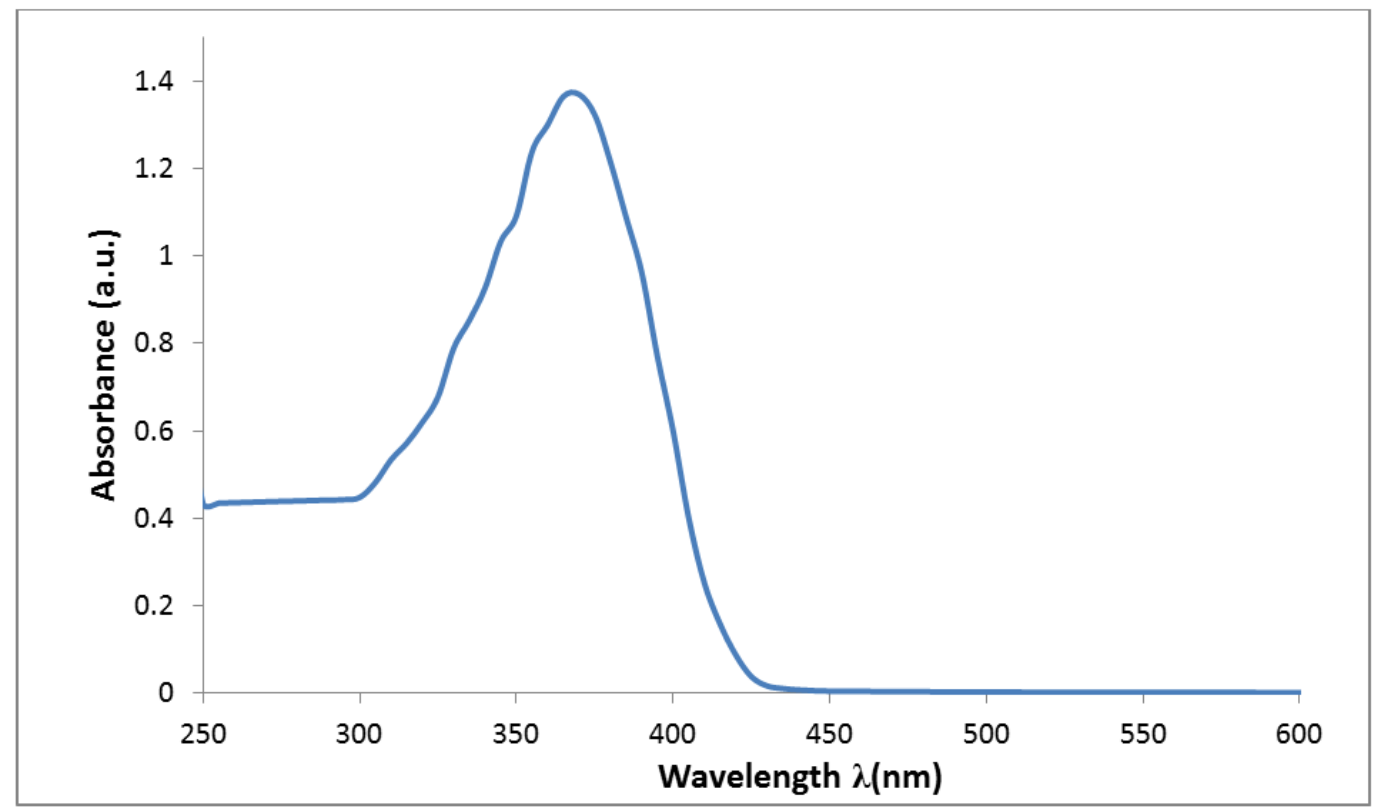

Figure 8. Experimental electronic spectrum for Bis (2-hydroxy benzylidene) benzidine.

\subsection{Fluorescence Characteristics}

The fluorescence spectrum of Bis (2-hydroxy benzylidene) benzidine in tetrahydrofuran solvent at the concentration of $2 \times 10^{-5} \mathrm{M}$ at $\mathrm{pH}(6)$ and room temperature shown in Figure (9). From this figure displays a production peak at $553 \mathrm{~nm}$ and excited at $400 \mathrm{~nm}$. The fluorescence amount was a good, this is because of prepared aromatic compound usually contain of interconnected aromatic circle structure of 2hydroxy benzylidene attached to the central structure, conjoining numerous ring structures increase the concentration of states and lets for a lower band gap substantial, letting electronic HOMO/LUMO excitation at lesser energies [20].

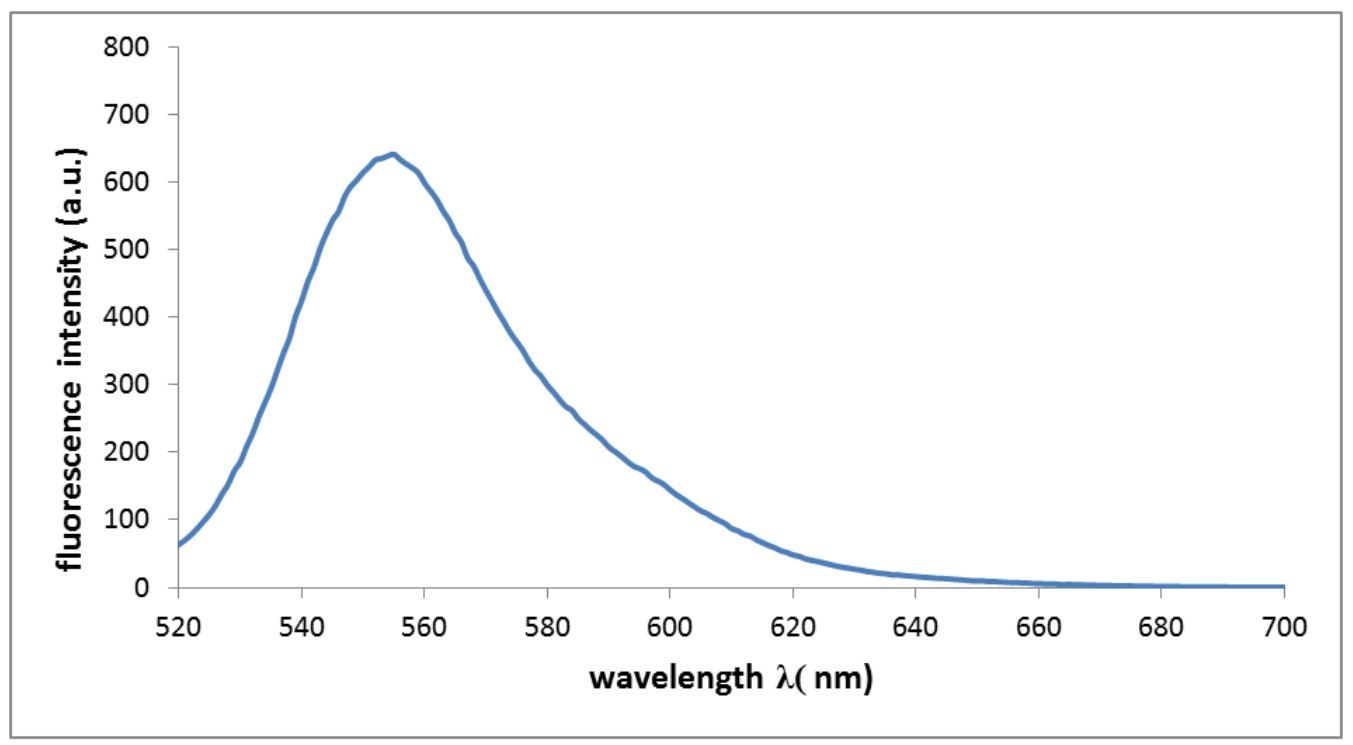

Figure 9. Fluorescence emission Spectrum of Bis (2-hydroxy benzylidene) benzidine.

\subsubsection{Effect of pH on Fluorescence Spectrum}

The intensity of Aggregation Induced Enhanced Emission (AIEE) of the System influence really by the $\mathrm{PH}$ of the medium [17]. The effect of acidic on the spectrum of $N, N^{\prime}$ Bis (2-hydroxy benzylidene) benzidine was examined by $\mathrm{NaOH}$ and $\mathrm{HCl}$ in aqueous can be seen in Figures $(10,11)$. Bis (2-hydroxy benzylidene) benzidine can exist in the next forms of different $\mathrm{pH}$ : (i) $\mathrm{pH}$ (5 to 7) High form (ii) $\mathrm{pH}$ (8 to
10) Low form and (iii) at $\mathrm{pH}$ (2 to 5) middle forms. The fluorescence intensity upsurges with increasing of $\mathrm{pH}$ (2 to $5)$. The intensity constant up to $\mathrm{pH}(5-7)$. The acidic domain was suitable for the stability of the compound name. Finally, it decreases up to $\mathrm{pH}=10$. The finest value for the fluorescence amount of PH (6), this mean that quantum yield was very good at this point. 


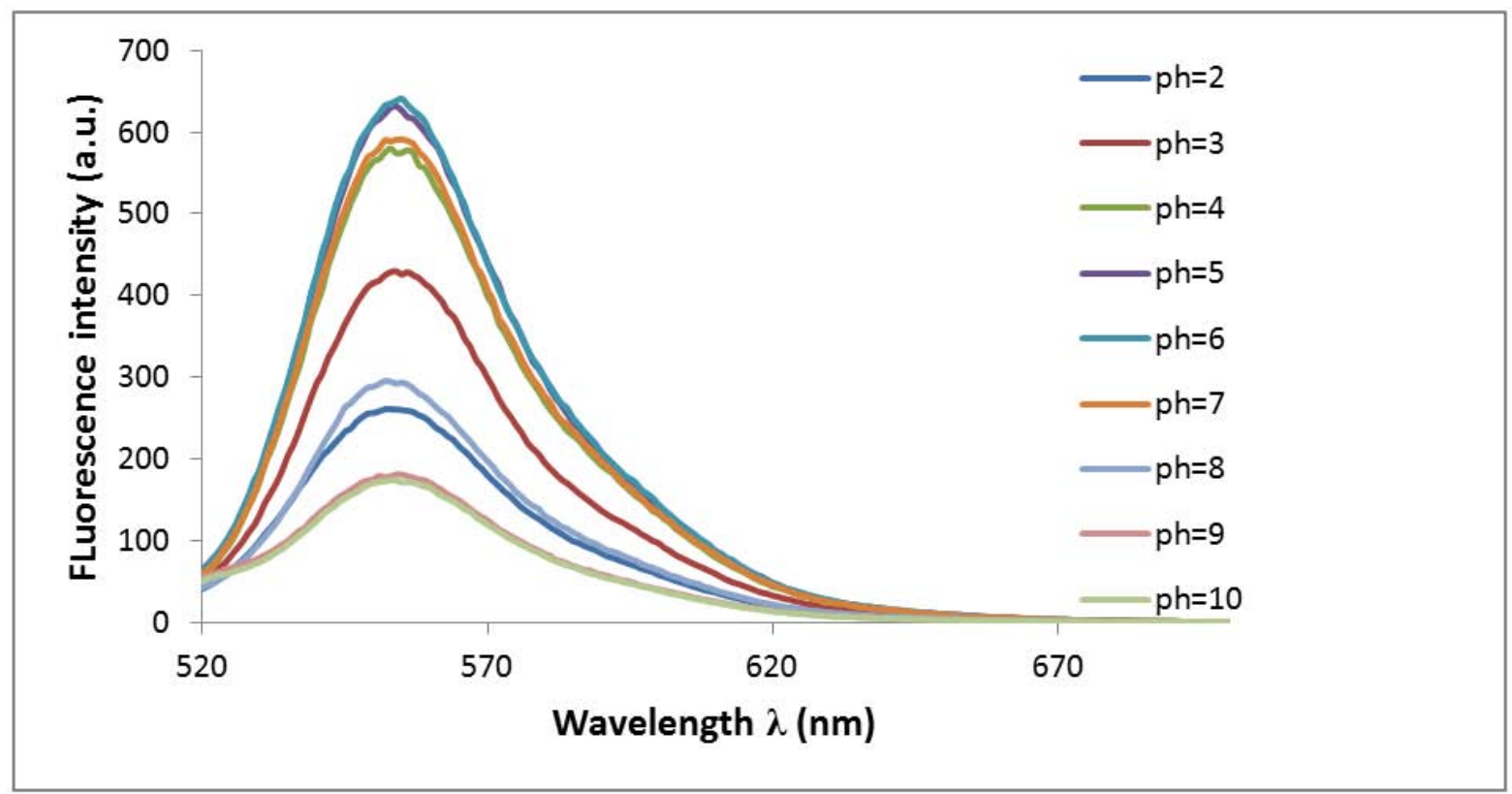

Figure 10. Effect of pH of fluorescence intensity of Bis (2-hydroxy benzylidene) benzidine.

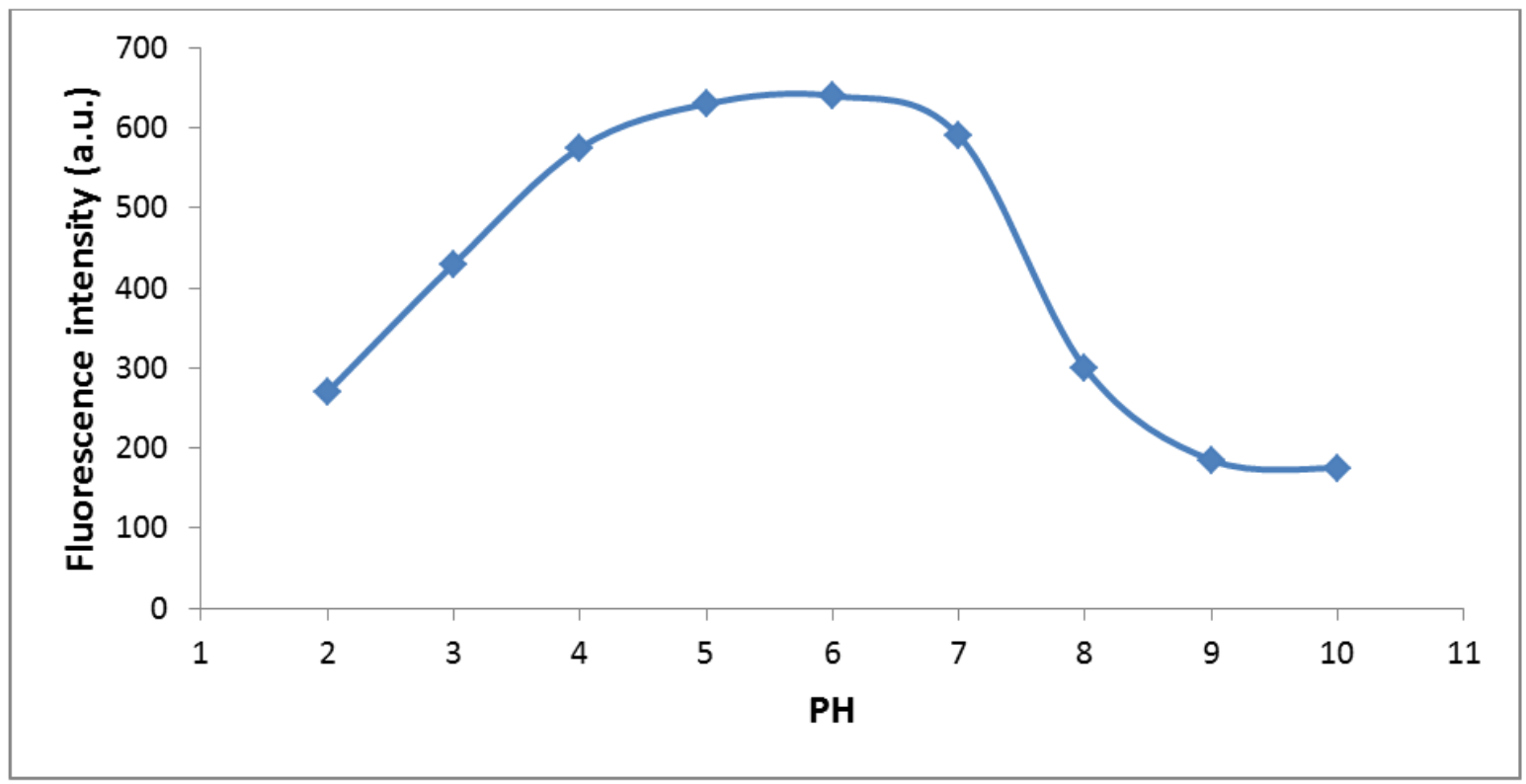

Figure 11. Effect of pH of fluorescence intensity of bis (2-hydroxy benzylidene) benzidine.

\subsubsection{Effect of Solvent on Fluorescence Spectral Properties}

In Table (2) determined the best emission and excitation wavelengths of $\mathrm{N}, \mathrm{N}^{\prime}$ Bis (2-hydroxy benzylidene) benzidinein with different solvents. According to the results, Bis (2-hydroxy benzylidene) benzidinein has the maximum emission in THF solvent. The Stoke's shift $\left(\Delta \lambda_{\mathrm{ST}}\right)$ in all using solvents was studied as in the literature [21] and can be seen in Table (1). The higher Stoke's shift provisions very little background signals and allows the usage of the cmpound in the construction of a fluorescence sensors. Bis (2-hydroxy benzylidene) benzidinein in THF solution had higher Stoke's shift with the value of $128 \mathrm{~nm}$, in comparison with the other solvents as seen in Table (2). This indicates that the usage of
Bis (2-hydroxy benzylidene) benzidinein as fluorophore is useful in THF solution. The emission spectra of Bis (2hydroxy benzylidene) benzidine in different solvents shown in Figure (12).

Table 2. Fluorescence spectra related data of Bis (2-hydroxy benzylidene) benzidinein.

\begin{tabular}{lllll}
\hline Solvent & $\lambda \boldsymbol{m a x}(\mathbf{E x})$ & $\lambda \max (\mathbf{E m})$ & $\mathbf{I}(\mathbf{E m})$ & $\Delta \lambda_{\text {ST }}$ \\
\hline THF & 400 & 553 & 640 & 153 \\
CDCL3 & 428 & 532 & 575 & 104 \\
Toluene & 429 & 534 & 610 & 105 \\
Acetone & 427 & 529 & 270 & 102 \\
\hline
\end{tabular}




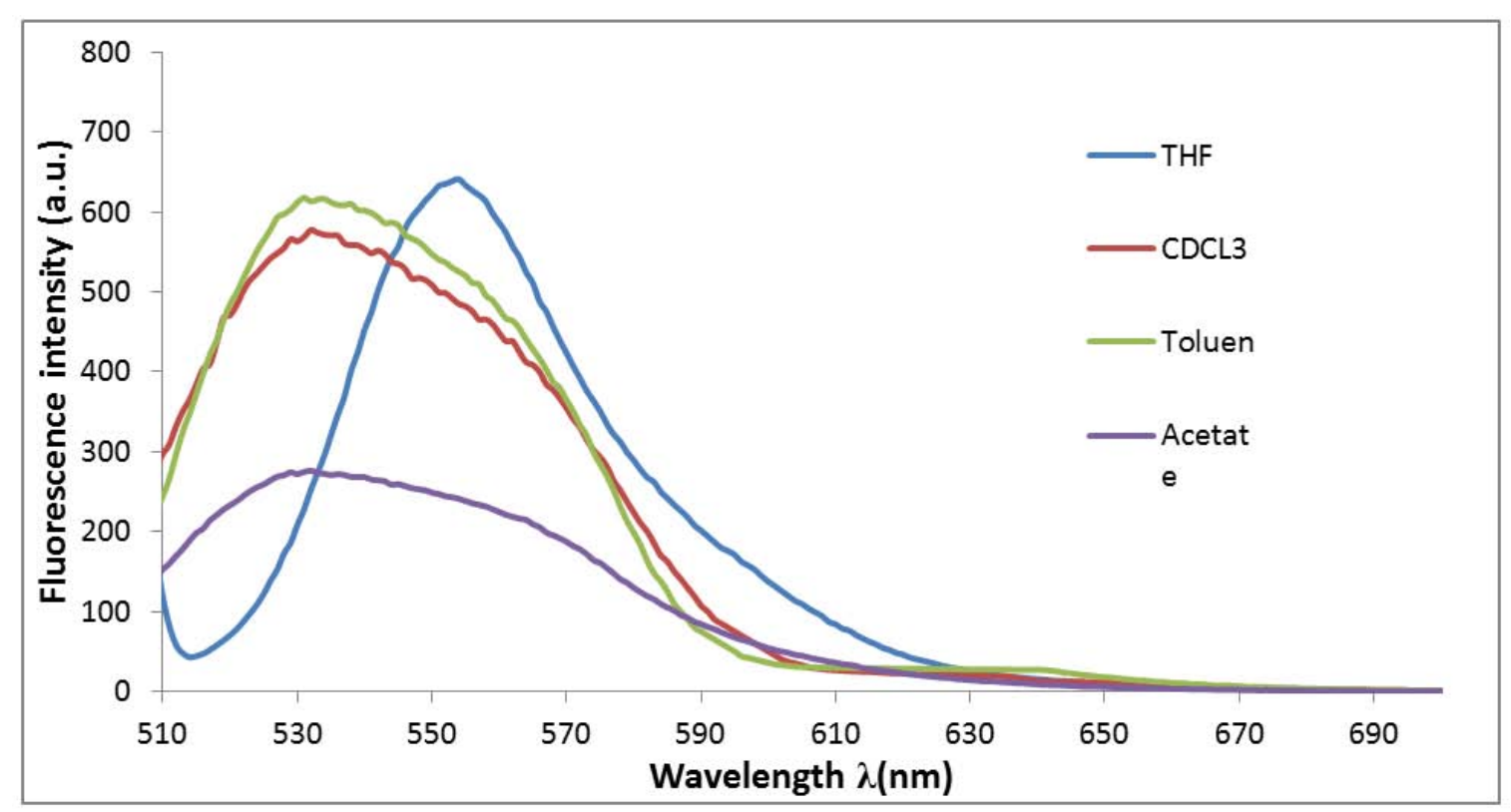

Figure 12. Emission spectra of Bis (2-hydroxy benzylidene) benzidinein in different solvents.

\section{Conclusion}

The new N, N' Bis (2-Hydroxy Benzylidene) Benzidine was synthesized. Uses the fluorescence spectrum, FT-IR, absorbance spectrum, and NMR techniques to study Characterizations of the title compound. Theoretical results of FT-IR, UV-vis, and NMR uses a DFT/B3LYP way with 6$311 \mathrm{G}(\mathrm{d}, \mathrm{p})$ basis set. The fluorescence intensity of the title compound in different solvents was defining. Stoke's shift values in the different solvents presented that THF was the finest solvent. It was strong the effect of $\mathrm{pH}$ on the Fluorescence intensity, the finest value for Fluorescence intensity of $\mathrm{pH}$ (5 to 7). This result of the $\mathrm{PH}$ value will increase quantum efficiency. Fluorescence intensities of $\mathrm{N}$, $\mathrm{N}^{\prime}$ Bis (2-Hydroxy Benzylidene) Benzidine in several solvents were studied. Stoke's shift values in the several solvents showed that THF was the finest solvent to use N, N' Bis (2-Hydroxy Benzylidene) Benzidine as fluorescent probes.

\section{Acknowledgements}

Authors are indebted to the backup by the ALMustansiriyah University College of Science, Physics and chemistry Department, especially, Mrs Beyader Fadhil Abbas.

\section{References}

[1] A. Barbieri, G. Accorsi, N. Armaroli. Luminescent complexes beyond the platinum group: the $\mathrm{d} 10$ avenue. Chem. Commun. (2008). 2185-2193.

[2] X. Q. Chen, X. Z. Tian, I. Shin, J. Yoon. A "turn-on" fluorescent probe for hypochlorous acid: convenient synthesis, good sensing. Chem. Soc. Rev. 40, (2011). 4783.

[3] Guoqiang Yang, Shayu Li, Shuangqing Wang1, Rui Hu, Jiao Feng, Yi Li, and Yan Qian. Novel fluorescent probes based on intramolecular charge- and proton-transfer compounds Pure Appl. Chem., Vol. 85, No. 7, (2013). pp. 1465-1478.

[4] bT. L. YANG, aW. W. QIN, aZ. F. XIAO, and aW. S. LIU, Synthesis and Infrared and Fluorescence Spectra of Europium and Terbium Complexes with a Novel Bis-Schiff Base Ligand Derived from 2,6-Diaminopyridine and Indole-2carbaldehyde. Chem. Pap. 59 (1) (2005). 17-20.

[5] Takuya Terai \& Tetsuo Nagano Pflügers Small-molecule fluorophores and fluorescent probes for bioimaging. Archiv European Journal of Physiology, Volume 465, Issue 3, (2013). pp 347-359.

[6] E. Szajdzinska-Pietek, M. Wolszczak and A. Plonka, S. J. Schlick. Fluorescence Studies of Self-Assembly in Aqueous Solutions of Poly (ethylene-co-methacrylic acid) Ionomers. J. Am. Chem. Soc, 120, (1998). 4215-4221.

[7] J. Y. Ye, M. T. Myaing, T. B. Norris, T. P. Thomas and J. R. BakerBiosensing based on two-photon fluorescence measurements through optical fibers. Optics Letters, 27, (2002) 1412-1414.

[8] C. S. Mitsiades, N. S. Mitsiades, T. T. Bronson, D. Chauhan, N. Munshi, S. P. Treon, C. A. Maxwell, L. Pilarski, T. Hideshima, R. M. Hoffman and K. C. Anderson. Fluorescence imaging of multiple myeloma cells in a clinically relevant SCID/NOD in vivo model: biologic and clinical implications. Cancer Res, 63, (2003). 6689.

[9] David W. Fink And Walter R. KoehlerpH Effects on fluorescenceof umbelliferone. Analytical Chemistry, Vol. 42, No. 9,. (1970). 990-993.

[10] C. Peng, P. Y. Ayala, H. B. Schlegel, M. J. Frisch, Using redundant internal coordinates to optimiz equilibrium geometries and transition states, J. Comput. Chem. 17 (1), 1996, 49-56. 
[11] M. J. Frisch, J. A. Pople, J. S. Binkley,, " Self- Consistent molecular orbital methods25: supplementary function for Gaussian basis sets," J. Chem. Phys., 80 (7), 1984, 32653269 .

[12] R. Ditchfield, "Self-consistent perturbation theory of diamagnetism. I. A Gauge-Invariant LCAO (Linear Combination of Atomic Orbitals) method for NMR chemical shifts," Mol. Phys., 27 (1974), 789-807.

[13] Z. Dong, X. Le, P. Zhou, C. Dong, J. Ma, .An "off-on-off" flu- orescent probe for the sequential detection of $\mathrm{Zn} 2+$ and hydrogensulfide in aqueous solution, New J. Chem. 38, (2014) 1802-1808.

[14] Geddes, C. D. et al., Metal-Enhanced Fluorescence (MEF) Due to Silver Colloids on a Planar Surface: Potential Applications of Indocyanine Green to in Vivo Imaging, J. Phys. Chem. A. 107, (2003). 3443- 3449.

[15] Aslan, K., Gryczynski, I., Malicka, J., Lakowicz, J. R. Geddes, C. D.,. Metal-Enhanced Fluorescence: An Emerging Trend in Biotechnology, Curr. Opin. Biotechnol. 16, (2005) $55-62$.

[16] Silverstein R. M., Webster F. X. Spectroscopic Identification of Organic Compound, sixth Ed., John Willey \& Sons, New York, 1998

[17] Mehmet Yıldırım \& İsmet Kaya)" Synthesis of a Novel Fluorescent Schiff Base as a Possible $\mathrm{Cu}$ (II)Ion Selective Sensor" J Fluoresc, 20: (2010)771-777.

[18] Nagabalasubramanian P. B., Periandy S., Mohan S., Govindarajan M. "FTIR and FT Raman spectra, vibrational assignments, ab initio, DFT and normal coordinate analysis of $\alpha, \alpha$ dichlorotoluene," Spectrochim. Acta, Vol. 73A (2), (2009). p.p. $277-280$

[19] Nasrin Soltani, Hosein Salavati, Nahid Rasouli, Mehrnoosh Paziresh, A. Moghadasi." Electrochemical and Quantum Chemical Calculations of Two Schiff Bases as Inhibitor for Mild Steel Corrosion in Hydrochloric Acid Solution" IRAN J ANAL CHEM 2,. (2015) 22- 35 / 23.

[20] R. Yuste, "Fluorescence microscopy today," Nat Meth, vol. 2, (2005). pp. 902-904, 12.

[21] Oter O, Ertekin K, Kılınçarslan R, Ulusoy M, Çetinkaya B. Photo characterization of a novel fluorescent Schiff base and investigation of its utility as an optical $\mathrm{Fe}+3$ sensor in PVC matrix. Dye Pigments 74: (2007) 730-735. 